\title{
Gastric Adenocarcinoma at the Sites of Gastric Sarcoidosis: A Case Report
}

\author{
Mohamed Modar Abidian ${ }^{\mathrm{a}, \mathrm{c}}$, Obada Tabbaa ${ }^{\mathrm{a}}$, Leen Al Saleh ${ }^{\mathrm{a}}$, Muhamad Alhaj Moustafa ${ }^{\mathrm{a}}$, \\ Rama Hritani ${ }^{\mathrm{a}}$, Deborah Topol ${ }^{\mathrm{a}}$, Mark C. Mattar ${ }^{\mathrm{b}}$
}

\begin{abstract}
Sarcoidosis is a systemic disease characterized by the formation of non-caseating granulomas in multiple organs with lungs being the most commonly involved. Gastrointestinal sarcoidosis is a rare entity. The relationship between sarcoidosis and malignancy was addressed in the past with no conclusive results. Herein, we present a case of a 48-year-old African American man with a history of gastric sarcoidosis, who was found to have a poorly differentiated gastric adenocarcinoma at the same site of the sarcoidosis.
\end{abstract}

Keywords: Sarcoidosis; Gastric cancer; Adenocarcinoma; Association; Risk

\section{Introduction}

Gastric cancer used to be one of the most common cancers worldwide in the last few decades [1].The incidence rate has been falling remarkably in the USA [2,3]. This decline could be attributed to appropriate management of the gastric cancer risk factors like Helicobacter pylori infection as well as dietary and environmental factors. The majority of gastric cancers are adenocarcinoma. Known risk factors of gastric adenocarcinoma include age, H. pylori infection, smoking, and intake of salty and smoked food which is common in East Asia [1]. Sarcoidosis as a factor risk for developing gastric cancer was not confirmed [4]. Multiple studies have failed to establish a steady association between sarcoidosis and malignancy. The meta-analysis by Bonifazi et al (2015) was able to demonstrate a moderately significant association between sarcoidosis and malignancy [5]. Such association could be ex-

Manuscript submitted April 18, 2019, accepted April 29, 2019

aDepartment of Internal Medicine, Georgetown University MedStar Washington Hospital Center, Washington, DC, USA

bDivision of Gastroenterology, Medstar Georgetown University Hospital, Washington, DC, USA

${ }^{\mathrm{c} C o r r e s p o n d i n g ~ A u t h o r: ~ M o h a m e d ~ M o d a r ~ A b i d i a n, ~ D e p a r t m e n t ~ o f ~ I n t e r n a l ~}$ Medicine, Georgetown University MedStar Washington Hospital Center, Washington, DC 20007, USA. Email: mmabidian@gmail.com

doi: https://doi.org/10.14740/jmc3302 plained by sarcoidosis chronic granulomatous inflammation that would predispose to malignancy. Herein, we present a case of gastric adenocarcinoma developed at the site of gastric sarcoidosis.

\section{Case Report}

A 48-year-old African American man had a medical history of hepatic and renal sarcoidosis that was diagnosed several years ago by biopsy of both organs. As part of his sarcoidosis workup, he underwent both esophagogastroduodenoscopy (EGD) and colonoscopy. The EGD showed nodular mucosa in the gastric antrum and biopsies from those lesions revealed non-necrotizing granulomas consistent with sarcoidosis. Colonoscopy gross evaluation was unremarkable and biopsies from the terminal ileum were negative for granulomas. As part of the follow-up workup, a computed tomography (CT) scan of the abdomen 5 years later showed enlarged liver with nodular morphology consistent with cirrhosis, but without discrete liver lesions. Ten years later, the patient presented to our hospital with 1-month history of epigastric pain and weight loss. Physical exam revealed mild epigastric tenderness along with mild ascites. Complete blood count (CBC) was unremarkable. Basic metabolic panel (BMP) showed chronic elevation of blood urea nitrogen (BUN) and creatinine levels consistent with end-stage kidney disease pattern. Liver function tests (LFTs) were only significant for mild elevation of aspartate aminotransferase (AST) with albumin levels of $2.4 \mathrm{~g} / \mathrm{dL}$. $H$. pylori stool antigen was negative. Chronic hepatitis panel labs including viral hepatitis, autoimmune hepatitis, Wilson disease, and primary biliary cirrhosis were all unremarkable. Abdominal CT scan of the abdomen showed a large $(7.2 \times 5.6$ $\mathrm{cm})$ circumferential mass in the gastric antrum, invading the muscle wall and causing partial obstruction of the gastric outlet (Fig. 1). Nodular cirrhotic liver morphology with multiple hypodense lesions in the liver suggestive of metastatic disease was also seen (Fig. 2).

EGD revealed a large, ulcerated, circumferential fungating mass in the gastric antrum with near total occlusion of gastric pylorus resulting in partial gastric outlet obstruction (Fig. 3 ). Biopsies from that lesion confirmed the diagnosis of poorly differentiated invasive adenocarcinoma that was negative for H. pylori infection (Fig. 4). Subsequent ultrasound-guided core biopsies from the hypodense liver lesions confirmed the 


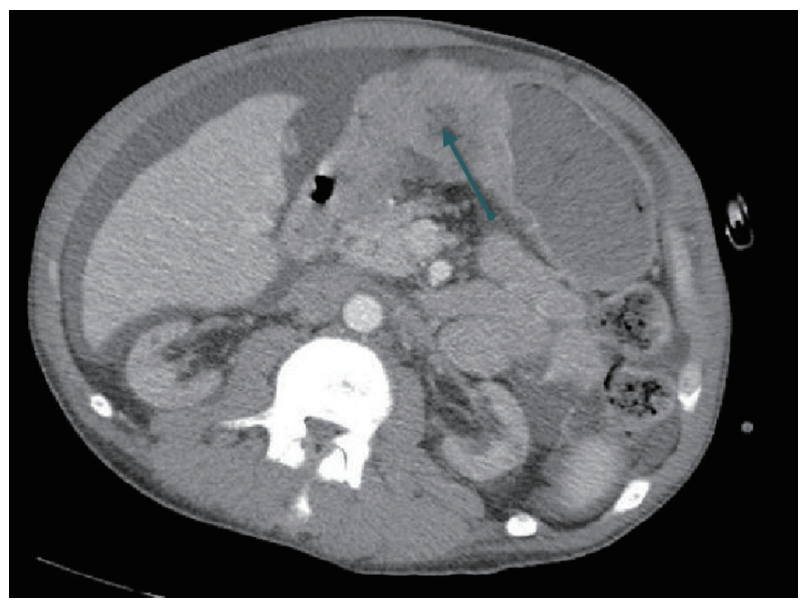

Figure 1. CT abdomen shows an antral gastric mass (arrow).

diagnosis of poorly differentiated metastatic gastric adenocarcinoma.

The patient clinical and nutritional status deteriorated due to intolerance of oral intake in the setting of partial gastric outlet obstruction. Gastrostomy and jejunostomy feeding tubes were contraindicated because of the underlying ascites. The patient developed anaerobic bacterial peritonitis thought to be related to the peritoneal dialysis access. The patient did not receive any chemotherapy due to his poor functional status and ongoing infections. He died in the intensive care unit 1 month after hospital admission.

\section{Discussion}

Gastrointestinal sarcoidosis represents up to $10 \%$ of all sarcoidosis cases with gastric antrum being the most common involved location [1]. In the USA, sarcoidosis is more common in the African American population who tend to develop a more

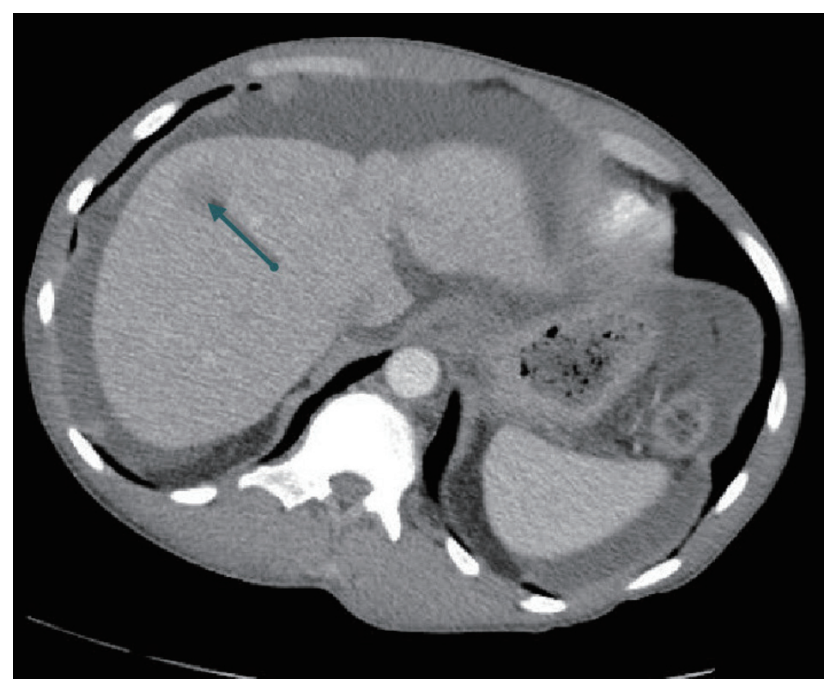

Figure 2. Same CT abdomen shows a metastatic hypodense liver lesion (arrow).

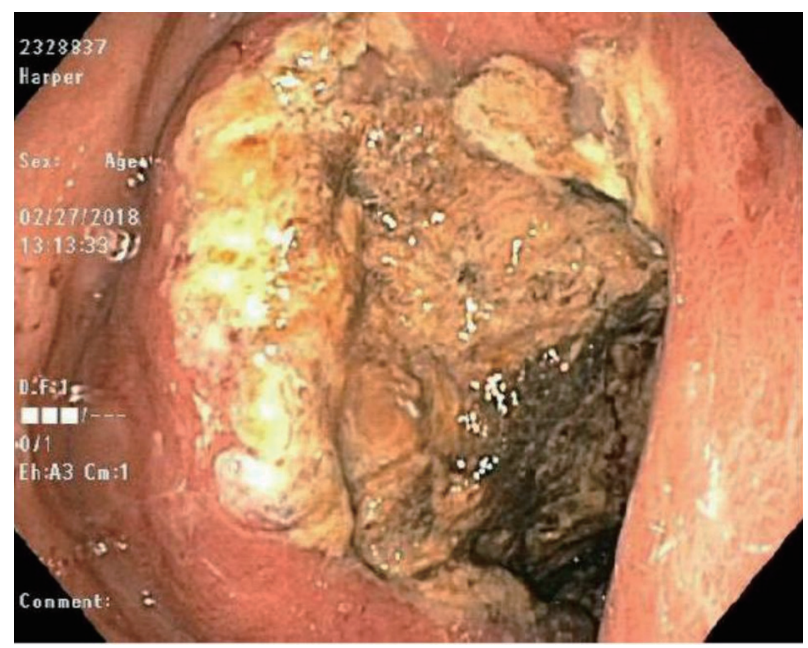

Figure 3. EGD shows an ulcerated mass in the stomach antrum with partial gastric outlet obstruction.

severe pattern of the disease compared to other populations [6]. In general, gastric sarcoidosis is asymptomatic most of the time. Clinical signs and symptoms are vague and non-specific including abdominal pain, nausea, vomiting, weight loss, and upper gastrointestinal bleeding [7]. EGD evaluation of the gastric mucosa would reveal different morphologic patterns like ulcerations, gastritis, diffuse erythematous friable mucosa, or nodular lesions that narrow the gastric lumen [7]. Sarcoidosis is characterized by the formation of non-caseating granuloma in the involved tissues. Biopsies and histopathologic examination of the suspected lesions are necessary to confirm the diagnosis and rule out other diseases like H. pylori gastritis, tuberculosis, and fungal infections [8]. In terms of treatment, corticosteroids are indicated when the disease is symptomatic with documented response rate up to $66 \%$ [6]. Symptomatic and endoscopic resolution with the steroid therapy has been documented without clear evidence of histopathologic regres-

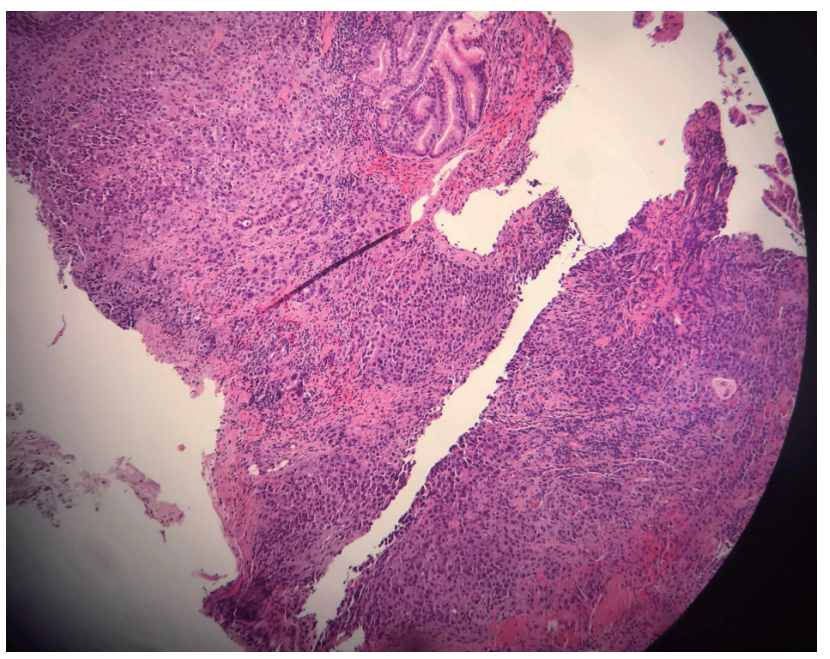

Figure 4. Histopathologic specimen of gastric antrum with H\&E staining shows poorly differentiated histopathologic pattern of adenocarcinoma. H\&E: hematoxylin and eosin. 
sion [8].

The association between sarcoidosis and malignancy was addressed in many retrospective studies with inconclusive results. An overall excess risk was found in few studies, although the results varied considerably, and data on selected cancer sites were inconsistent. On the other hand, some studies failed to demonstrate such association [9-11]. Results from a systematic review and meta-analysis published in 2015 showed a moderate, yet significant, association between sarcoidosis and malignancy. The review included 16 original studies with more than 25,000 patients from various countries worldwide. The relative risk (RR) of all invasive cancers was $1.19(95 \%$ confidence interval (CI), 1.07 - 1.32) with slight higher risk for skin, hematopoietic, and upper gastrointestinal tract (RR, $1.73 ; 95 \%$ CI, 1.07 - 2.79). Mechanisms that would explain the association between sarcoidosis and malignancy include sarcoidosis chronic inflammation, immune dysfunction, and genetic susceptibility to both cancer and autoimmune diseases. Moreover, it is understood that the gastrointestinal mucosa is rich in lymphoid tissue which might serve as a favorable target for the sarcoidosis which in turn predisposes to lymphoproliferative diseases $[5,10]$.

We are reporting a case of antral gastric adenocarcinoma developed at the site of antral gastric sarcoidosis years later. Even though our patient had a history of smoking, the carcinogenic role of sarcoidosis cannot be ignored based on the abovementioned facts. Our aim from reporting this case is to draw clinical researchers' attention to fully evaluate the relationship between sarcoidosis and cancer development. This could be addressed by conducting further studies focusing on identifying cancer predictors of patients with sarcoidosis such as disease severity, organ involvement and response to therapy. Moreover, such studies can attribute to a better understanding of carcinogenesis mechanism in patients with sarcoidosis for future development of cancer surveillance programs.

\section{Acknowledgments}

None to declare.

\section{Financial Disclosure}

None to declare.

\section{Conflict of Interest}

None to declare.

\section{Informed Consent}

Not applicable.

\section{Author Contributions}

MMA wrote the whole manuscript and obtained the endoscopy and pathology pictures; OT, LAS, MAM, RH, DT and MCM helped with organizing the manuscript structure and reviewing the article.

\section{References}

1. Karimi P, Islami F, Anandasabapathy S, Freedman ND, Kamangar F. Gastric cancer: descriptive epidemiology, risk factors, screening, and prevention. Cancer Epidemiol Biomarkers Prev. 2014;23(5):700-713.

2. Haenszel W. Variation in incidence of and mortality from stomach cancer, with particular reference to the United States. J Natl Cancer Inst. 1958;21(2):213-262.

3. Fitzsimmons D, Osmond C, George S, Johnson CD. Trends in stomach and pancreatic cancer incidence and mortality in England and Wales, 1951-2000. Br J Surg. 2007;94(9):1162-1171.

4. Ji J, Shu X, Li X, Sundquist K, Sundquist J, Hemminki K. Cancer risk in hospitalized sarcoidosis patients: a followup study in Sweden. Ann Oncol. 2009;20(6):1121-1126.

5. Bonifazi M, Bravi F, Gasparini S, La Vecchia C, Gabrielli A, Wells AU, Renzoni EA. Sarcoidosis and cancer risk: systematic review and meta-analysis of observational studies. Chest. 2015;147(3):778-791.

6. Afshar K, BoydKing A, Sharma OP, Shigemitsu H. Gastric sarcoidosis and review of the literature. J Natl Med Assoc. 2010;102(5):419-422.

7. Friedman M, Ali MA, Borum ML. Gastric sarcoidosis: a case report and review of the literature. South Med J. 2007;100(3):301-303.

8. Liang DB, Price JC, Ahmed H, Farmer N, Montgomery EA, Giday SA. Gastric sarcoidosis: case report and literature review. J Natl Med Assoc. 2010;102(4):348-351.

9. Cohen PR, Kurzrock R. Sarcoidosis and malignancy. Clin Dermatol. 2007;25(3):326-333.

10. McGhee JR, Fujihashi K. Inside the mucosal immune system. PLoS Biol. 2012;10(9):e1001397.

11. Le Jeune I, Gribbin J, West J, Smith C, Cullinan P, Hubbard R. The incidence of cancer in patients with idiopathic pulmonary fibrosis and sarcoidosis in the UK. Respir Med. 2007;101(12):2534-2540. 